\title{
Chapter
}

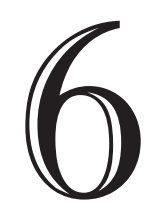

\section{Structural characterization and immunogenicity in wildtype and immune tolerant mice of degraded recombinant human interferon alpha2b}

Suzanne Hermeling, Liliana Aranha, J. Mirjam A. Damen', Monique Slijper ${ }^{1}$, Huub Schellekens, Daan J.A. Crommelin, Wim Jiskoot

${ }^{1}$ Department of Biomolecular Mass Spectrometry, Utrecht Institute for Pharmaceutical Sciences (UIPS) and Bijvoet Center for Biomolecular research 


\begin{abstract}
Purpose. To study the influence of protein structure on the immunogenicity in wildtype and immune tolerant mice of well-characterized degradation products of recombinant human interferon alpha2b (rhIFNo2b).

Methods. RhIFN $\alpha 2 b$ was degraded by metal catalyzed oxidation (M), crosslinking with glutaraldehyde $(\mathrm{G})$, oxidation with hydrogen peroxide $(\mathrm{H})$ and incubation in a boiling water bath (B). The products were characterized with UV absorption, circular dichroism and fluorescence spectroscopy, gel permeation chromatography, reversed-phase HPLC, SDS-PAGE, Western blotting and mass spectrometry. The immunogenicity of the products was evaluated in wildtype mice and in transgenic mice immune tolerant for hIFNo2. Serum antibodies were detected by ELISA or surface plasmon resonance.
\end{abstract}

Results. M-rhIFN $\alpha 2 b$ contained covalently aggregated rhIFN $\alpha 2 b$ with three methionines partly oxidized to methionine sulfoxides. G-rhIFNa2b contained covalent aggregates and did not show changes in secondary structure. H-rhIFNo2b was only chemically changed with four partly oxidized methionines. B-rhIFN $\alpha 2 b$ was largely unfolded and heavily aggregated. Native (N) rhIFNo2b was immunogenic in the wildtype mice but not in the transgenic mice, showing that the latter were immune tolerant for rhIFN $\alpha 2 b$. The antirhIFN $\alpha 2 b$ antibody levels in the wildtype mice depended on the degradation

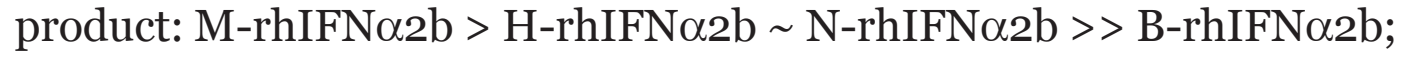
G-rhIFN $\alpha 2 b$ did not induce anti-rhIFN $\alpha 2 b$ antibodies. In the transgenic mice, only M-rhIFN $\alpha 2 b$ could break the immune tolerance.

Conclusions. RhIFN $22 \mathrm{~b}$ immunogenicity is related to its structural integrity. Moreover, the immunogenicity of aggregated rhIFN $\alpha 2 b$ depends on the structure and orientation of the constituent protein molecules and/or on the aggregate size. 


\section{Introduction}

Nearly all therapeutic proteins induce antibodies. In many cases the incidence and the clinical consequences are limited, but the development of a number of promising new biopharmaceuticals has been stopped because of immunogenicity problems. The development of antibodies in patients may be associated with life-threatening side effects and limits the clinical use of biopharmaceuticals for the treatment of chronic and serious diseases for which there is no alternative effective medical treatment (1).

There are two immunological mechanisms by which these antibodies are induced. A classical, relatively fast immune reaction occurs after treatment with a therapeutic protein from non-human origin, e.g. streptokinase or asparaginase. Patients react to these foreign proteins as to vaccines. A second mechanism for the induction of antibodies is based on breaking B-cell tolerance, which in general takes many months. This may occur in patients who are treated with products which are homologues of human proteins. The exact mechanisms by which therapeutic proteins break tolerance is unknown, although impurities and aggregates are known to be important factors (2-8). Impurities may act as danger signals initiating a response to self-antigens and aggregates may present the self-antigens in a repetitive array form, which is supposed to activate B-cells without T-cell help (9-11).

Although several predictive approaches have been advocated, clinical trials are still the only way to establish the immunogenicity of protein drugs. Immune tolerant transgenic mice are the most useful tool in studying the immunogenicity of therapeutic proteins. They have been used to evaluate whether sequence variations of existing therapeutic proteins as insulin induced new epitopes (12) and to study the breaking of tolerance by aggregates (4).

We have extended these studies and have evaluated whether such models can also be used for more detailed studies on the relation between structure of the therapeutic proteins and the breaking of B-cell tolerance. As a model protein we have selected recombinant human interferon alpha2b (rhIFN $\alpha 2 b)$. Transgenic immune tolerant animals carrying the human IFNo2 gene are available (4). Moreover, this protein is used in the treatment of a variety of malignancies and viral diseases (13) and has a record of relative high immunogenicity (14). The induction of antibodies is associated with the loss of efficacy (14). Marketed rhIFN $\alpha 2$ products contain either rhIFN $\alpha 2 a$ or rhIFN $\alpha 2 b$, which differ in one amino acid which does not affect immunogenicity (15). A review of the production and purification process has been published (5). Lyophilization of rhIFN $\alpha 2 b$ formulations containing human serum albumin and improper storage conditions have been shown to 
lead to the formation of aggregates $(5,16)$. The level of anti-rhIFN $\alpha 2$ antibodies is related to the presence of aggregates $(5,15)$.

We present here data on the relation between the structure of rhIFN $\alpha 2 b$, modified by different chemical and physical treatments, and its immunogenicity in wildtype and transgenic immune tolerant mice.

\section{Materials and methods}

\section{Degradation of rhIFN $\alpha 2 b$}

RhIFN $\alpha 2 b$ (a gift from AlfaWassermann, Bologna, Italy) was degraded in four different ways. All samples were in $10 \mathrm{mM}$ sodium phosphate buffer, pH 7.4 (PB).

To oxidize the rhIFN $\alpha 2 b$ it was treated with hydrogen peroxide $\left(\mathrm{H}_{2} \mathrm{O}_{2}\right)$, according to the European Pharmacopeia (17), by incubating rhIFN $\alpha 2 b$ $(1 \mathrm{mg} / \mathrm{ml})$ with $0.005 \%(\mathrm{v} / \mathrm{v}) \mathrm{H}_{2} \mathrm{O}_{2}$ at $37^{\circ} \mathrm{C}$ for 20 hours. The oxidation was stopped by the addition of $12 \mathrm{mg}$ methionine per $\mathrm{ml}$ followed by incubation at room temperature for one hour.

The metal catalyzed oxidation was achieved by incubating rhIFN $\alpha 2 b$ $(300 \mu \mathrm{g} / \mathrm{ml})$ with $4 \mathrm{mM}$ ascorbic acid and $0.04 \mathrm{mM} \mathrm{CuCl}_{2}$ for three hours at room temperature according to $\mathrm{Li}$ et al (18). The reaction was stopped by adding $100 \mathrm{mM}$ EDTA to a final concentration of $1 \mathrm{mM}$.

The rhIFN $\alpha 2 b$ was also crosslinked with glutaraldehyde according to Braun et al (4): incubation of rhIFN $\alpha 2 b(200 \mu \mathrm{g} / \mathrm{ml})$ with $0.04 \%$ (v/v) glutaraldehyde at room temperature for two minutes, followed by 20 hours incubation after adding sodium borohydride in a final concentration of $1.2 \mathrm{mM}$.

RhIFN $\alpha 2 b$ was also modified by incubating $300 \mu \mathrm{g} / \mathrm{ml}$ in a boiling waterbath for ten minutes.

The oxidized and glutaraldehyde samples were dialyzed against $10 \mathrm{mM}$ PB before use. All samples were stored at $-20^{\circ} \mathrm{C}$.

\section{Protein concentration}

Protein concentrations were measured with a modified Lowry method (19). Bovine serum albumin (BSA) (Sigma-Aldrich, Zwijndrecht, The Netherlands) was used as a standard. 
Characterization of rhIFN $\alpha 2 b$

Unless stated otherwise, all dilutions were in $10 \mathrm{mM} \mathrm{PB}$ and all analyses were performed without prior filtration.

\section{UV spectroscopy}

UV spectra (200-450 $\mathrm{nm})$ of the samples $(200 \mu \mathrm{g} / \mathrm{ml})$ were recorded on a Perkin Elmer Lambda 2 UV/VIS spectrophotometer in quartz cuvettes with a pathlength of $1 \mathrm{~cm}$. Ten $\mathrm{mM} P B$ was used as a blank.

\section{Circular dichroism (CD) spectroscopy}

CD spectra were recorded from $260-180 \mathrm{~nm}$ at $25{ }^{\circ} \mathrm{C}$ in $0.5-\mathrm{mm}$ quartz cuvettes with a dual-beam DSM 1000 CD spectrophotometer (On-Line Instrument Systems, Bogart, Georgia, USA). The subtractive double-grating monochromator was equipped with a fixed disk, holographic gratings (2400 lines/mm, blaze wavelength $230 \mathrm{~nm}$ ), and 1.24-mm slits. The protein concentration was $100 \mu \mathrm{g} / \mathrm{ml}$. Each measurement was the average of at least ten repeated scans (step resolution $1 \mathrm{~nm}, 1 \mathrm{~s}$ each step) from which the corresponding buffer spectrum was subtracted. The CD signals were converted to delta molar extinction based on a mean residual weight of 117. The curves were smoothed with GraphPad Prism 4.02 for Windows (GraphPad Software, San Diego, California, USA), by taking the weighted average of nine points.

\section{Fluorescence spectroscopy}

Fluorescence emission spectra (280 - $450 \mathrm{~nm}$; 1-nm steps) of $55 \mu \mathrm{g} / \mathrm{ml}$ samples were measured in 1-cm quartz cuvettes in a Fluorolog III fluorimeter at $25{ }^{\circ} \mathrm{C}$ while stirring. Excitation was at $295 \mathrm{~nm}$. Slits were set at $5 \mathrm{~nm}$. Integration time per data point was $0.1 \mathrm{~s}$ and the average of ten scans was taken. The buffer signal was subtracted.

\section{Dynamic light scattering (DLS)}

Samples $(200 \mu \mathrm{g} / \mathrm{ml})$ were analyzed with DLS to obtain an average diameter of the particles $\left(\mathrm{Z}_{\text {ave }}\right)$ and their polydispersity at an angle of $90^{\circ}$. A Malvern CGS-3 apparatus equipped with $\mathrm{He}-\mathrm{Ne}(633 \mathrm{~nm})$ JDS Uniphase laser, an optical fiber based detector, and ALV/LSE-5003 correlator was used. 


\section{Gel permeation chromatography (GPC)}

Samples $(100 \mu \mathrm{g} / \mathrm{ml})$ were analyzed with a Superdex $20010 / 300$ GL column (Amersham, Roosendaal, The Netherlands) using a mobile phase of $50 \mathrm{mM}$ PB and $200 \mathrm{mM}$ sodium chloride, filtered through a $0.2 \mu \mathrm{m}$ filter prior to use, at a flow rate of $0.50 \mathrm{ml} / \mathrm{min}$ by a Waters 2695 controller equipped with an autosampler. Chromatograms were recorded with a photodiode array detector (model 2996, Waters). The column was calibrated by analyzing protein standards obtained from Sigma-Aldrich (Zwijndrecht, The Netherlands) with known molecular weights (i.e. thyroglobulin, BSA, ovalbumin, $\alpha$-chymotrypsin and myoglobin).

\section{Sodium dodecyl sulfate polyacrylamide gel electrophoresis (SDS-PAGE)}

The gels consisted of a separating gel containing $15 \%(\mathrm{w} / \mathrm{v})$ acrylamide and $0.1 \%(\mathrm{w} / \mathrm{v})$ SDS, and a stacking gel containing $5 \%(\mathrm{w} / \mathrm{v})$ acrylamide and $0.1 \%$ $(\mathrm{w} / \mathrm{v})$ SDS. Gels of $0.75 \mathrm{~mm}$ thickness were run under reducing (sample buffer containing $5 \%(\mathrm{v} / \mathrm{v}) \beta$-mercaptoethanol) and non-reducing conditions at $200 \mathrm{~V}$ at room temperature. The electrophoresis buffer was $25 \mathrm{mM}$ tris (hydroxymethyl) aminomethane, $192 \mathrm{mM}$ glycine and 0.1\% (w/v) SDS. Gel electrophoresis was done with a Biorad Protean III system (Biorad, Veenendaal, The Netherlands). Samples analyzed under reducing conditions were boiled for five minutes before application to the gel. A low range molecular weight standard (Biorad) was included on the gel for determination of molecular weight.

\section{Western blotting}

SDS-PAGE gels were blotted onto a nitrocellulose sheet with a Scie-Plas semi-dry blotter (Scie-Plas, UK). Blots were blocked overnight at $4{ }^{\circ} \mathrm{C}$ with $1 \%(\mathrm{w} / \mathrm{v})$ non-fat milk powder in $0.005 \%(\mathrm{w} / \mathrm{v})$ Tween 20 in phosphate buffered saline (PBS) with constant orbital shaking. After washing with $0.005 \%(\mathrm{w} / \mathrm{v})$ Tween 20 in PBS and with water, the blots were incubated with polyclonal rabbit anti-rhIFN $\alpha 2 b$ serum in $0.1 \%(\mathrm{w} / \mathrm{v})$ non-fat milk powder in $0.005 \%(w / v)$ Tween 20 in PBS for one hour at room temperature with constant orbital shaking. Blots were washed with $0.005 \%$ (w/v) Tween 20 in PBS and with water. Blots were incubated with peroxidase labeled goat antirabbit IgG (Sigma) in $0.1 \%(\mathrm{w} / \mathrm{v})$ non-fat milk powder in $0.005 \%(\mathrm{w} / \mathrm{v})$ Tween 20 in PBS for one hour at room temperature with constant orbital shaking. Blots were washed with $0.005 \%(\mathrm{w} / \mathrm{v})$ Tween 20 in PBS and with water and incubated in a solution of 4-chloro-1-naphtol (Sigma) in methanol (15\% (v/v)), 
water and $\mathrm{H}_{2} \mathrm{O}_{2}(0.015 \%(\mathrm{v} / \mathrm{v}))$. After color development the blots were stored overnight in the dark in water to increase the intensity of the bands.

\section{Reversed-phase high pressure liquid chromatography (RP-HPLC)}

A ProSphere $\mathrm{C} 18$ (300 $\AA ; 5 \mu \mathrm{m})$ was used in combination with an All-Guard C18 guard column (Alltech, Breda, The Netherlands). The column was equilibrated with $45 \%(\mathrm{w} / \mathrm{w})$ acetonitrile in $0.2 \%(\mathrm{v} / \mathrm{w})$ trifluoroacetic acid (TFA) for at least 30 minutes. After application of the sample (50 $\mu$, $100 \mu \mathrm{g} / \mathrm{ml})$ a gradient of $45-50 \%(\mathrm{w} / \mathrm{w})$ acetonitrile in $0.2 \%(\mathrm{v} / \mathrm{w})$ TFA in 25 minutes was applied to the column. The mobile phase was delivered to the column at a flow rate of $1 \mathrm{ml} / \mathrm{min}$ by a Waters 2695 controller equipped with an integrated autosampler. Chromatograms were recorded with a photodiode array detector (model 2996, Waters).

\section{Mass spectrometry}

Mass spectrometric measurements were performed on an ESI-ToF (LCT) mass spectrometer (Micromass, UK) to obtain the total mass of the protein. The instrument was equipped with a Z-nano-electrospray source (Micromass) operating in the positive ionization mode. Nanoflow electrospray needles were made from borosilicate glass capillaries (Kwik-Fil, World Precision Instruments, Sarasota, Florida, USA) on a P-97 puller (Sutter instruments, Novato, California, USA). The needles were coated with a thin gold layer using a Scancoat Six sputter coater (Edwards Laboratories, Milpitas, California, USA). To remove sodium ions, samples were dialyzed first overnight against $50 \mathrm{mM}$ ammonium acetate.

To analyze and identify the oxidation sites in the oxidized rhIFN $\alpha 2 b$ samples matrix-assisted laser desorption ionization mass spectrometry (MALDI ToF-ToF, AB 4700 Proteomics analyzer, Applied Biosystems) was used. This instrument is equipped with a $200 \mathrm{~Hz} \mathrm{Nd}$ :YAG laser operating at $355 \mathrm{~nm}$. Experiments were performed in a reflectron positive ion mode using delayed extraction. Typically, 2250 shots per spectrum were acquired in the MS mode.

The oxidized samples and native rhIFN $\alpha 2 b$ were reduced with dithiothreitol (DTT) and treated with iodoacetamide to protect the cysteines. After iodoacetamide treatment the samples were dialyzed against $10 \mathrm{mM} \mathrm{PB}$ and digested with trypsin (Sigma) for 20 hours at $37^{\circ} \mathrm{C}$. The trypsin to protein ratio was 1 to $50(\mathrm{w} / \mathrm{w})$. The digested protein was mixed with $5 \mathrm{mg} / \mathrm{ml}$ $\alpha$-cyano-4-hydroxycinnamic acid in $50 \%(\mathrm{v} / \mathrm{v})$ acetonitrile and $0.1 \%(\mathrm{v} / \mathrm{v}) \mathrm{TFA}$ 
and spotted on the MALDI plate. The obtained mass spectra were compared with theoretical peptide masses obtained after theoretical digestion of the protein and oxidation of the methionines using the 'proteomics tools' utility of the ExPASy web site (20).

\section{Immunogenicity}

\section{Animal experiment}

The animal experiment was approved by the Institutional Ethical Committee. Wildtype (FVB/N) mice were obtained from Charles River laboratories. Transgenic mice were bred at the Central Laboratory Animal Institute. Food (Hope Farms, Woerden, The Netherlands) and water (acidified) were available ad libitum.

To test the immunogenicity of the samples wildtype or transgenic mice (five per group) were injected intraperitoneally (i.p.) with $10 \mu$ g protein on days 0-4, 7-11 and 14-18. Blood was collected from the vena saphena on days $0,7,14$, before injection and on day 21. The blood samples were incubated on ice for two hours. Sera were collected after centrifugation and stored at $-20^{\circ} \mathrm{C}$.

To make sure that the immune tolerance of the transgenic mice was specific for hIFN $\alpha 2$, five wildtype and five transgenic mice were immunized with $10 \mu \mathrm{g}$ ovalbumin using the same injection protocol as described above. The presence of anti-ovalbumin antibodies in the serum was determined as described below.

\section{Serum analysis by ELISA}

Sera were analyzed by ELISA for the presence of antibodies against native rhIFN $\alpha 2 b$ and against the corresponding degraded sample, or against ovalbumin. Microlon 96-well plates (Greiner, Alphen aan de Rijn, The Netherlands) were incubated with $100 \mu \mathrm{l}$ per well of a solution $(2 \mu \mathrm{g} / \mathrm{ml})$ of

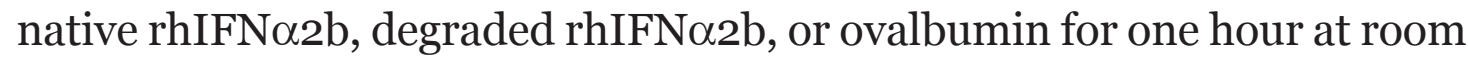
temperature with constant orbital shaking. Then the wells were drained and washed four times with $300 \mu \mathrm{l}$ wash buffer (0.1\% (w/v) Tween 20 in PBS). After washing the wells were carefully tapped dry on a tissue. Wells were blocked by incubating with $200 \mu \mathrm{l} 2 \%$ (w/v) BSA in PBS for one hour at room temperature with constant orbital shaking. The plates were drained and washed four times with $300 \mu \mathrm{l}$ wash buffer. After the last wash, wells were carefully tapped dry on a tissue. Serum dilutions (1:100) were added to the wells and the plates were incubated for one hour at room temperature with constant orbital shaking. The plates were washed four times with $300 \mu \mathrm{l}$ wash 
buffer. After the last wash, wells were carefully tapped dry on a tissue. Peroxidase labeled goat anti-mouse IgG (Sigma) was added to the wells and the plates were incubated for one hour at room temperature with constant orbital shaking. Plates were drained and washed four times with $300 \mu \mathrm{l}$ wash buffer and twice with $300 \mu \mathrm{lBBS}$. After the last wash, wells were carefully tapped dry on a tissue. ABTS (2,2' -azinobis-(3-ethylbenzothiazoline-6-sulfonic acid)) substrate (Roche, Almere, The Netherlands) was added and absorbance was recorded after 30 minutes of incubation, on a Novapath microplate reader (Biorad) at a wavelength of $415 \mathrm{~nm}$ and a reference wavelength of $490 \mathrm{~nm}$. During all incubation steps the plates were covered.

Sera were arbitrarily defined positive when the absorbance value of the 1:100 dilution of the sera minus the background was three times higher than the average absorbance value of the pretreatment sera minus the background.

To determine the antibody titer of the positive sera, the sera were added to the plates, coated with native rhIFN $\alpha 2 b$ or ovalbumin, in serial dilutions (starting from 1:10). The other steps of the ELISA procedure were as described above and the absorbance values were plotted against log dilution. Curves were fitted with a sigmoidal curve (GraphPad Prism version 4.02 for Windows). The dilution needed to obtain $50 \%$ of the maximum absorbance was taken as the titer of the serum.

\section{Serum analysis by surface plasmon resonance}

The sera of the mice treated with boiled rhIFN $\alpha 2 b$ were analyzed by surface plasmon resonance (SPR) with a Biacore (Biacore, Uppsala, Sweden). Native and boiled rhIFN $\alpha 2 b$ were coupled via an amine coupling to two different flow cells of a CM5 sensor chip (Biacore). The flow cells were washed with HBS-EP buffer (10 mM HEPES, pH 7.4, 0.15 M sodium chloride, 3 mM EDTA, $0.005 \%$ (w/v) Tween 20). After the coupling, $10 \mu \mathrm{l}$ serum (diluted 10 fold with HBS-EP buffer) of the wildtype mice (pooled per time point) or of the transgenic mice (pooled per time point) were injected on the two different flow cells of the $\mathrm{CM}_{5}$ sensor chip. After the injection the amount of bound material was determined by measuring the increase in relative response units. Complete dissociation of the sera from the sensor chips was obtained by washing the flow cells with a $1.5 \mathrm{M}$ glycine solution. 


\section{Results}

Characterization of degraded rhIFN $\alpha 2 b$

All samples were optically clear and colorless, without visible aggregation or precipitation. Only after repeated freeze-thawing, boiled rhIFN $\alpha 2 b$ showed visible precipitation.

\section{UV spectroscopy}

The UV spectra of proteins can give valuable information about the presence of aggregates in the solution. The metal catalyzed oxidized and boiled rhIFN $\alpha 2 b$ preparations showed significant optical densities at $350 \mathrm{~nm}$ (table 1) and above, which indicates the presence of aggregates (21). In boiled rhIFN $\alpha 2 b$, metal catalyzed oxidized rhIFN $\alpha 2 b$ and glutaraldehyde treated rhIFN $\alpha 2 b$ a decrease in $\mathrm{A}_{280} / \mathrm{A}_{260}$ ratio as compared to native rhIFN $\alpha 2 \mathrm{~b}$ was observed, also indicative for the presence of aggregates (21). The UV spectrum of $\mathrm{H}_{2} \mathrm{O}_{2}$ oxidized rhIFN $\alpha 2 b$ almost overlapped with native rhIFN $\alpha 2 b$ (data not shown).

Table 1 Characteristics of spectroscopy measurements of native and degraded rhIFN $\alpha 2 b$.

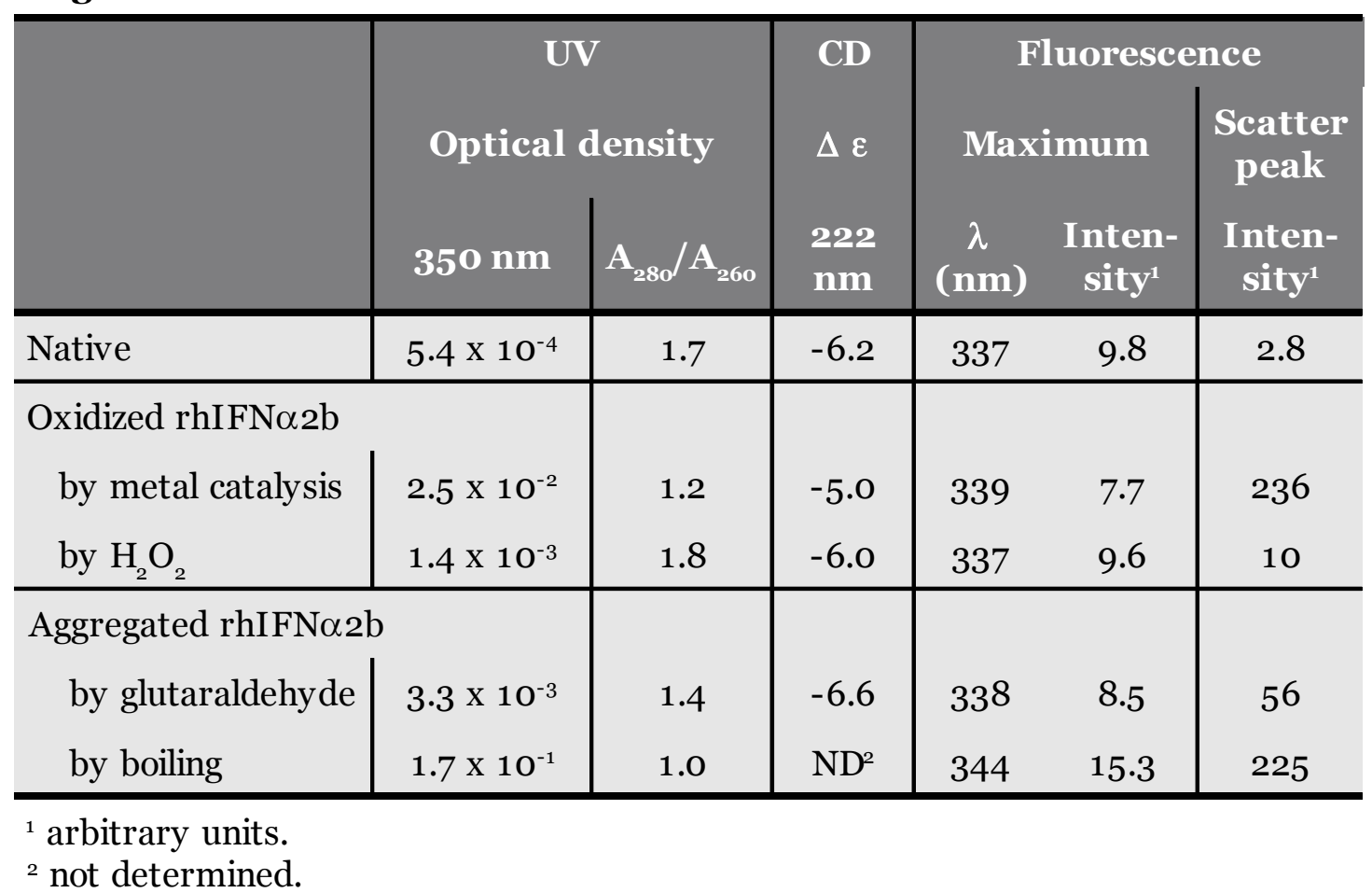




\section{CD spectroscopy}

Far-UV CD spectroscopy was used to determine the secondary structure of rhIFN $\alpha 2 b$, which contains five $\alpha$-helices (data not shown). Native rhIFN $\alpha 2 b$ gave a far-UV CD spectrum typical for an $\alpha$-helical protein. Metal catalyzed oxidized rhIFN $\alpha 2 b$ showed a small reduction of the intensity of the entire spectrum (but not the shape), indicating a small decrease in $\alpha$-helical content (table 1). Glutaraldehyde treated and $\mathrm{H}_{2} \mathrm{O}_{2}$ oxidized rhIFN $\alpha 2 b$ showed similar far-UV CD spectra as compared to native rhIFN $\alpha 2 b$. A far-UV CD spectrum of boiled rhIFN $\alpha 2 b$ could not be obtained, due to the presence of precipitate in the solution.

\section{Fluorescence spectroscopy}

Fluorescence spectroscopy, when performed with an excitation wavelength of $295 \mathrm{~nm}$, gives information about the environment of the two tryptophans in rhIFN $\alpha 2 b$ at positions 76 and 140. In addition, the scattering of the light (signal of $295 \mathrm{~nm}$ ) provides information on the presence of particles or aggregates in the solution.

Native rhIFN $\alpha 2 \mathrm{~b}$ showed an emission maximum at $337 \mathrm{~nm}$ and little scattering (table 1). The emission spectrum of metal catalyzed oxidized rhIFN $\alpha 2 b$ was slightly red-shifted, indicating a more hydrophilic environment of at least one of the tryptophans. The substantial increase in scatter intensity indicates the presence of aggregates. Glutaraldehyde treated rhIFN $\alpha 2 b$ showed also a slightly red-shifted maximum and an increased scatter peak, but less than metal catalyzed oxidized rhIFNa2b. The emission spectrum of $\mathrm{H}_{2} \mathrm{O}_{2}$ oxidized rhIFN $\alpha 2 b$ had the same characteristics as that of native rhIFN $\alpha 2 b$. The emission spectrum of boiled rhIFN $\alpha 2 b$ showed a large red-shift, indicating that the environment of the tryptophans had become more hydrophilic. The protein was, however, not completely unfolded, because in $6 \mathrm{M}$ guanidine chloride the emission maximum was at $356 \mathrm{~nm}$ (data not shown). The high intensity of the scatter peak of boiled rhIFN $\alpha 2 b$ was about the same as that of metal catalyzed oxidized rhIFN $\alpha 2 b$, pointing to extensive aggregation.

\section{$\underline{D L S}$}

Only the metal catalyzed oxidized and boiled rhIFNo2b samples scattered enough light to analyze the particle size by DLS. In the metal catalyzed oxidized solution the average diameter of the particles was ca. $0.7 \mu \mathrm{m}$, with a high polydispersity index (0.84), indicating the presence of aggregates 
heterogeneous in size. Boiled rhIFN $\alpha 2 b$ showed large (ca. 1-3 $\mu \mathrm{m}$ )

heterodisperse aggregates.

\section{$\underline{G P C}$}

GPC was used to analyze the samples for the presence of soluble aggregates (figure 1 and table 3). Native rhIFN $\alpha 2 \mathrm{~b}$ showed a peak of monomeric protein and a small dimer peak. Metal catalyzed oxidized rhIFN $\alpha 2 b$ contained clearly less monomer than native rhIFN $\alpha 2 b$, whereas dimers and some trimers had been formed. Also a small, broad peak of larger aggregates was present in this sample. Glutaraldehyde treated rhIFN $\alpha 2 b$ showed a huge decrease in monomer content, with a concomitant increase in di-, tri- and tetramer content and some bigger oligomers. $\mathrm{H}_{2} \mathrm{O}_{2}$ oxidized rhIFNa2b showed a profile similar to that of native rhIFN $\alpha 2 b$. Boiled rhIFN $\alpha 2 b$ did not show any peaks on GPC (data not shown), indicating that practically all protein molecules had formed insoluble aggregates.

\section{SDS-PAGE}

Formation of covalent aggregates was determined by comparing SDS-PAGE gels run under non-reducing and reducing conditions (figures $2 \mathrm{a}$ and $\mathrm{b}$,
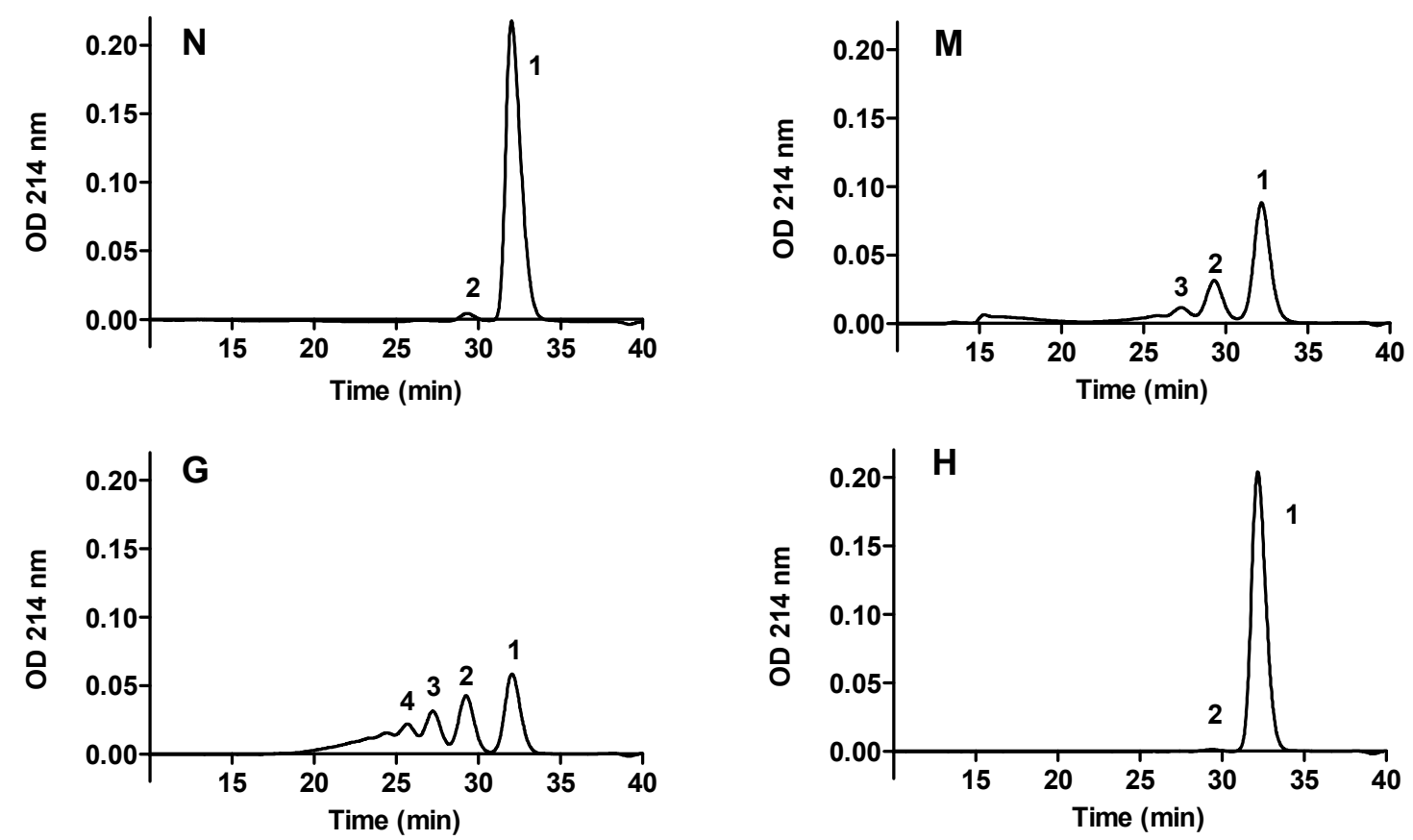

Figure 1 Gel permeation chromatograms of native rhIFN $\alpha 2 b(N)$ and degraded samples.

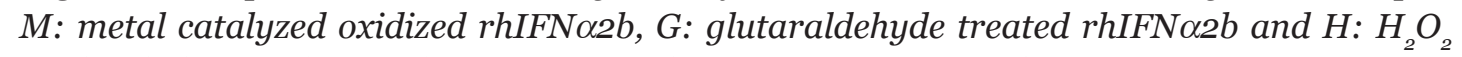
oxidized rhIFN $\alpha 2 b$. Numbers correspond to: 1: monomer; 2: dimer; 3: trimer; 4: tetramer. Masses were confirmed with a calibration curve obtained as described in the materials and methods section. 
respectively). Native and $\mathrm{H}_{2} \mathrm{O}_{2}$ oxidized rhIFN $\alpha 2 b$ showed a monomer band and a small dimer band under non-reducing conditions. The dimer band was nearly absent under reducing conditions, indicating that the dimer formation was primarily mediated by disulfide bonds. Also the multimeric bands of metal catalyzed oxidized rhIFN $\alpha 2 b$ seen under non-reducing conditions were substantially less intense under reducing conditions, pointing to disulfide bonds forming the multimers. Both gels showed similarly intense bands of aggregated protein for glutaraldehyde treated rhIFNo2b. These aggregates are apparently covalently linked via (non-reducible) glutaraldehyde crosslinks. The non-reduced sample of boiled rhIFN $\alpha 2 b$ contained dimers and larger multimers formed through disulfide bridges, as they were almost absent after reduction of the samples.

\section{Western blotting}

The reactivity of the individual bands on SDS-PAGE (non-reducing conditions) with polyclonal anti-rhIFN $\alpha 2 b$ serum was tested by Western blotting (figure 2c). As expected, the monomer of native rhIFN $\alpha 2 b$ reacted with the polyclonal antiserum. This was also true for the single band of $\mathrm{H}_{2} \mathrm{O}_{2}$ oxidized rhIFNo2b. Aggregates that were present in metal catalyzed oxidized
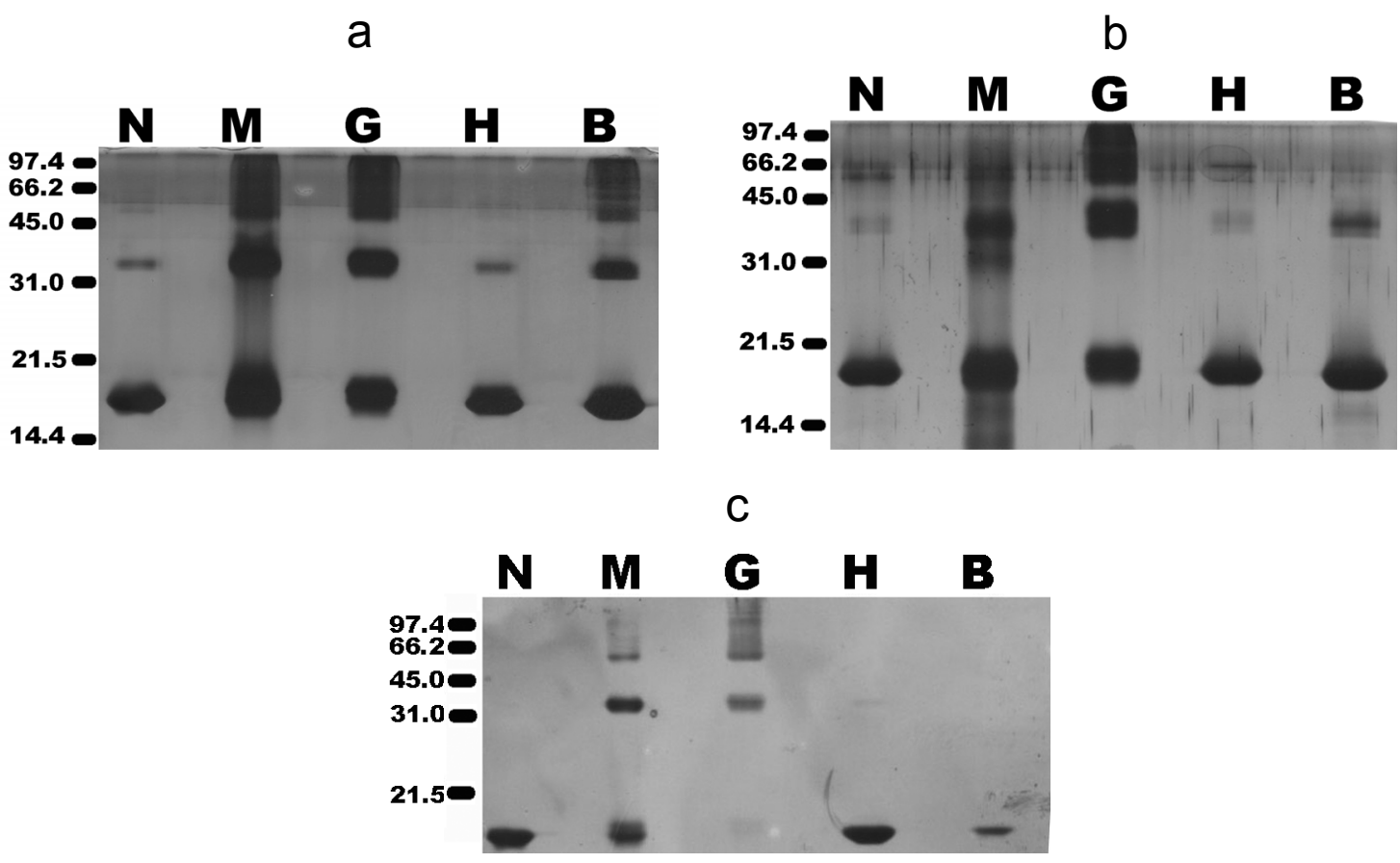

Figure 2 SDS-PAGE gels (a: non-reducing and b: reducing) and Western blot of a gel run under non-reducing conditions (c) of native and degraded rhIFN $22 b$. Numbers on the left represent band positions (in $\mathrm{kDa}$ ) of the molecular weight markers. Lane $N=$ native rhIFN $\alpha 2 b ; M=$ metal catalyzed oxidized rhIFN $\alpha 2 b ; G=$ glutaraldehyde treated rhIFN $\alpha 2 b ; \mathrm{H}_{2} \mathrm{H}_{2} \mathrm{O}_{2}$ oxidized rhIFN $\alpha 2 b$ and $\mathrm{B}=$ boiled rhIFN $\alpha 2 b$. 
rhIFN $\alpha 2 b$ and glutaraldehyde treated rhIFN $\alpha 2 b$ reacted with the antiserum as well. The dimers and larger aggregates in boiled rhIFNo2b did not react with the polyclonal antiserum. Possibly the antiserum did not recognize the aggregates or the amount of aggregates transferred onto the nitrocellulose sheet was too low to be detected by this method.

The monomer of glutaraldehyde treated rhIFN $\alpha 2 b$ did not react with the antiserum. Apparently the chemical treatment had not only crosslinked the protein molecules, but also destroyed the immuno-dominant epitopes in the remaining monomer.

\section{$\underline{R P-H P L C}$}

Only native and $\mathrm{H}_{2} \mathrm{O}_{2}$ oxidized rhIFN $\alpha 2 b$ showed peaks in the RP-HPLC chromatogram (data not shown). Metal catalyzed oxidized, glutaraldehyde treated and boiled rhIFN $\alpha 2 \mathrm{~b}$ did not show any peaks, probably because the aggregated rhIFN $\alpha 2 b$ was captured by the guard column and the amount of non-aggregated rhIFN $\alpha 2 b$ was too low to be detected with this method. However, GPC showed that metal catalyzed oxidized rhIFN $\alpha 2 b$ contained $43 \%$ of monomeric rhIFN $\alpha 2 b$ (table 3). Possible explanations for the apparent discrepancy between GPC and RP-HPLC are: (a) the solubility of metal catalyzed oxidized rhIFN $\alpha 2 b$ in the mobile phases used in RP-HPLC was lower than that of native rhIFNo2b; (b) the aqueous GPC solvent was capable of breaking apart non-covalent aggregates more easily than the organic RP-HPLC solvents; (c) a smaller amount of protein was applied on RP-HPLC than on GPC; (d) adsorption to the guard column present in RP-HPLC, or (e) a combination of the above possibilities.

Compared with native rhIFN $\alpha 2 b, \mathrm{H}_{2} \mathrm{O}_{2}$ oxidized rhIFN $\alpha 2 b$ showed an extra peak with a shorter retention time, representing oxidized rhIFN $\alpha 2 b$ (5). Based on the peak areas, about $60 \%$ of the protein was oxidized.

\section{Mass spectrometry}

In the electrospray mass spectrometer the protein becomes multiple charged, resulting in different $\mathrm{m} / \mathrm{z}$ peaks in the mass spectrum (22). From this so-called charge envelope the mass of the sample can be calculated. The base peak of native rhIFN $\alpha 2 \mathrm{~b}$ had a mass of 19,266 $\mathrm{Da}$ (corresponding to the theoretical value of 19,269 $\mathrm{Da}$ (23)). The base peaks of both oxidized rhIFN $\alpha 2 b$ preparations corresponded to a mass of 19,282 Da. The increase of 16 mass units confirms the addition of 1 oxygen atom, indicating that oxidation had occurred. The peak corresponding to non-oxidized rhIFN $\alpha 2 b$ was also 
Table 2 Ratios of oxidized to non-oxidized peptides determined by MALDI-ToF-ToF spectrometry.

\begin{tabular}{|c|c|c|c|}
\hline \multirow[b]{2}{*}{$\begin{array}{l}\text { Methionine } \\
\text { at position }\end{array}$} & \multicolumn{3}{|c|}{ Sample } \\
\hline & $\begin{array}{c}\text { Native } \\
\text { rhIFN } \alpha 2 b\end{array}$ & $\begin{array}{l}\text { Metal catalyzed } \\
\text { oxidized rhIFN } \alpha 2 b\end{array}$ & $\begin{array}{l}\mathrm{H}_{2} \mathrm{O}_{2} \text { oxidized } \\
\text { rhIFN } \alpha 2 b\end{array}$ \\
\hline 17 or $22^{1}$ & 0.09 & 0.40 & 1.28 \\
\hline 17 and 22 & 0.01 & 0.10 & 0.19 \\
\hline 60 & $\mathrm{O}^{2}$ & $\mathrm{ND}^{3}$ & ND \\
\hline 112 & o & ND & o \\
\hline 149 & 0.55 & 1.75 & 1.61 \\
\hline
\end{tabular}

${ }^{1}$ Only peptides containing both methionines were detected. These peptides were detected with 1 ( 17 or 22 ) or 2 (17 and 22) methionines oxidized. It was not possible to distinguish between the two methionines.

${ }^{2}$ Only peaks corresponding to peptides containing the oxidized methionine were detected.

${ }^{3} \mathrm{ND}$ : no peaks of peptides containing this methionine were detected, neither oxidized, nor non-oxidized.

visible, indicating that not all molecules were oxidized. No peaks in the mass-spectrum of glutaraldehyde treated rhIFN $\alpha 2 b$ could be obtained with this method; the sample was too heterogeneous in mass. Boiled rhIFNa2b had the same calculated mass as native rhIFNa2b. However, the charge envelope of the boiled sample contained more peaks and the average $\mathrm{m} / \mathrm{z}$ ratio was shifted to lower $\mathrm{m} / \mathrm{z}$ values, indicating that the boiled protein was unfolded (22). Aggregates were not detected with this method.

Table 2 shows the ratios of the peak intensities of peptides (obtained by tryptic digestion, see materials and methods) containing oxidized methionines over those of the corresponding non-oxidized peptides, as obtained with MALDI-ToF-ToF analysis. In native rhIFN $\alpha 2 b$ some oxidized peptides were detected. Methionines 17, 22 and 149 were shown to be oxidized in some of the protein molecules. In the oxidized samples, both metal catalyzed oxidized as well as $\mathrm{H}_{2} \mathrm{O}_{2}$ oxidized, the fraction of molecules containing these methionines oxidized had increased substantially as compared to native rhIFN $\alpha 2 b$, as is clear from the higher peak ratios of oxidized/non-oxidized peptides (see table 2). Peaks corresponding to peptides containing methionine 60, oxidized or non-oxidized, could not be detected for both oxidized samples. In addition, for metal catalyzed oxidized rhIFN $\alpha 2 b$ no peaks corresponding to peptides containing methionine 112 could be detected. The mass spectra showed no evidence of oxidized amino acid residues other than the methionines.

With MALDI-ToF-ToF of the peptide digests more oxidation sites were 


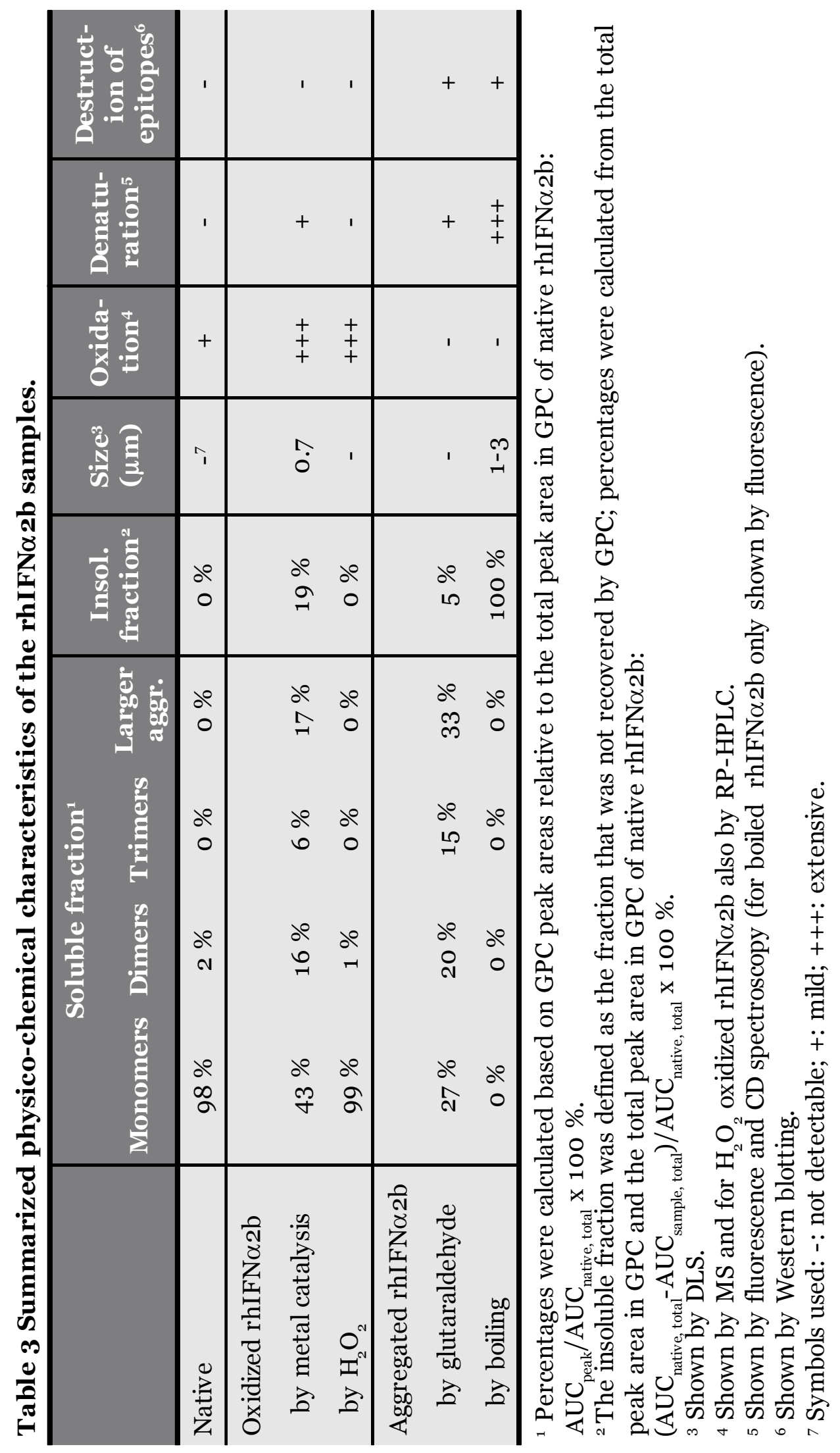


found than with ESI-ToF of the intact polypeptide chain. Probably the peak intensity of oxidation products with more than one oxidation site was too low to be resolved by ESI-ToF.

\section{Summary}

The main characteristics of the degraded samples, including the estimated amount of aggregated species, are summarized in table 3 .

\section{Immunogenicity}

Different immunization protocols (route of administration, dose, dosing schedule) were tested beforehand to optimize our animal model. We aimed for maximum and reproducible antibody levels for native rhIFN $\alpha 2 b$ in the wildtype mice. No matter which protocol was used, the transgenic mice never formed detectable antibodies against the native protein. This illustrates that they are immune tolerant for the native rhIFNo2b, indeed. These studies led to the immunization scheme mentioned in the materials and methods section.

To assure that the immune tolerance of the transgenic mice is specific for hIFN $\alpha 2$ and not due to a general immune suppression mechanism, we compared the antibody response against ovalbumin in the wildtype and the transgenic mice. Ovalbumin is foreign to both the wildtype and the transgenic mice, and should therefore elicit similar antibody responses. Figure 3 shows that the anti-ovalbumin IgG titers of the wildtype and the transgenic mice are comparable indeed, illustrating that the immune system of the transgenic mice is fully functional.

The anti-rhIFN $\alpha 2 b$ IgG titers of the wildtype mice immunized with native and degraded rhIFN $\alpha 2 \mathrm{~b}$ are shown in figure 4. Metal catalyzed oxidized and $\mathrm{H}_{2} \mathrm{O}_{2}$ oxidized rhIFN $\alpha 2 b$ induced antibodies to native rhIFN $\alpha 2 b$ in the

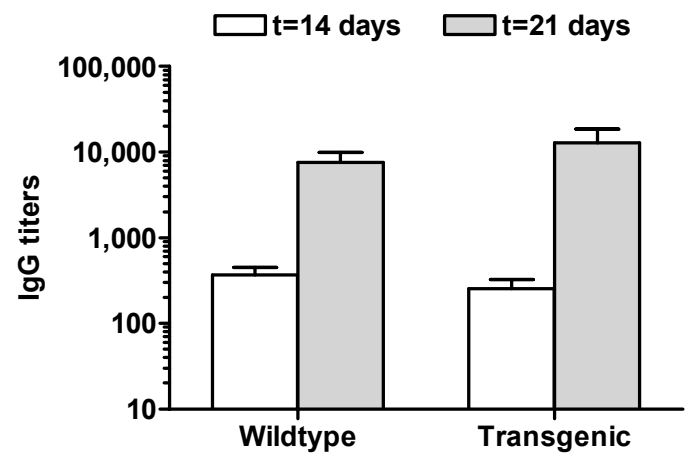

Figure 3 Anti-ovalbumin IgG titers in sera of wildtype and transgenic mice. Values represent average (+ SEM) titers. 
wildtype mice.

Only one of the wildtype mice treated with the boiled product produced antibodies to native rhIFN $\alpha 2 b$, while glutaraldehyde treated rhIFN $\alpha 2 b$ did not induce antibodies to native rhIFN $\alpha 2 b$ at all.

However, both the oxidized and glutaraldehyde treated rhIFN $\alpha 2 b$ preparations did induce antibodies in the wildtype mice to the respective modified material (figure $4 b$ ).

We failed to find an antibody response to native rhIFN $\alpha 2 b$ in the mice treated with boiled rhIFN $\alpha 2 b$. To exclude the possibility that this lack of response was due to the inability to coat ELISA plates with boiled protein, we also analyzed the sera with SPR. With this technology we picked up a weak antigen-antibody reaction, although it proved more difficult to immobilize boiled than native rhIFN $\alpha 2 b$ to the SPR chip. The boiled sample may have contained some native rhIFN $\alpha 2 b$, which was more efficiently coupled to the chip than boiled rhIFN $\alpha 2 b$. Therefore, we could not conclude from these results whether the antibodies were anti-rhIFN $\alpha 2 b$ or anti-boiled rhIFN $\alpha 2 b$.

Figure 5 shows the antibody response of the immune tolerant transgenic mice treated with the different rhIFN $\alpha 2 b$ 's. Only metal catalyzed oxidized rhIFN $\alpha 2 b$ was able to break the immune tolerance in the transgenic mice. The other degraded samples did not induce an antibody response, neither to native rhIFNa2b (figure 5), nor to the corresponding samples (data not shown). Also SPR failed to show antibodies against native or boiled rhIFN $\alpha 2 b$ in the sera of transgenic mice treated with boiled rhIFNo2b.
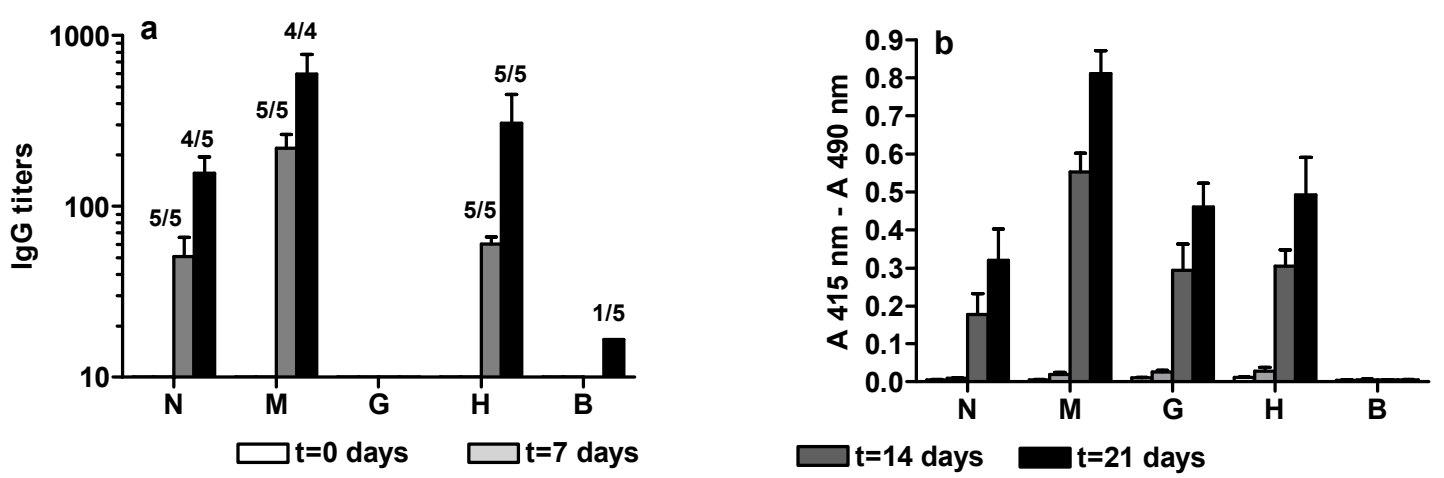

Figure 4 Anti-rhIFN $\alpha_{2} b$ IgG titers (a) and absorbance values of the 1:10o diluted (b) sera of wildtype mice treated with native $(N)$ and degraded rhIFN $\alpha 2 b$. The ELISA plate was coated with native rhIFN $22 b$ (a) or the corresponding test samples (b). M: metal

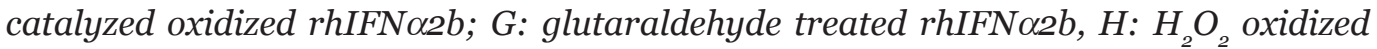
rhIFN $\alpha 2 b$ or B: boiled rhIFN $\alpha 2 b$. The values represent average (+SEM) titers of responding mice. The numbers above the bars (a) represent the number of responding mice out of the number of total mice. In the group mice receiving metal catalyzed oxidized rhIFN $22 b$ one mouse died at day 14 because of handling. 


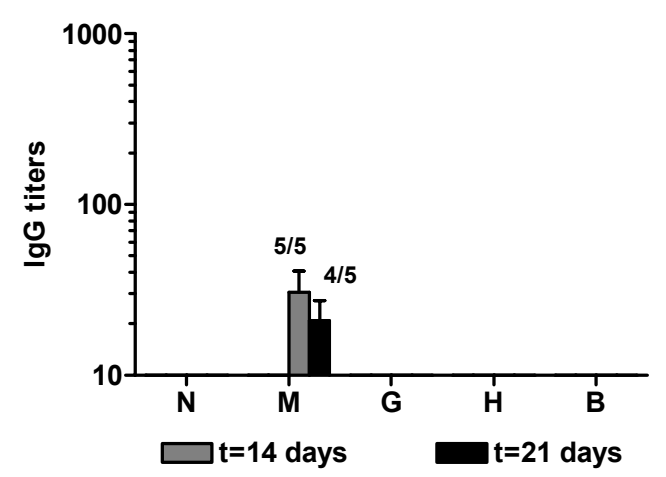

Figure 5 Anti-rhIFN $\alpha 2 b$ IgG titers in sera of transgenic mice treated with native $(N)$ and

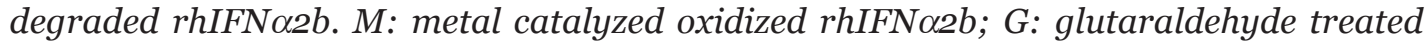
rhIFN $\alpha 2 b, \mathrm{H}_{2} \mathrm{H}_{2} \mathrm{O}_{2}$ oxidized rhIFN $\alpha 2 b$ or B: boiled rhIFN $\alpha 2 b$. The values represent average (+ SEM) titers of responding mice. The numbers above the bars represent the number of responding mice out of the number of total mice.

\section{Discussion}

Aggregates in therapeutic protein formulations are known to be important factors in inducing an antibody response (4-8). The immunological mechanism of this antibody induction is not completely understood. Aggregation may lead to conformational changes and the presentation of new epitopes. Aggregation may also lead to the type of multimeric epitope presentation which can break immune tolerance. Somehow the B-cell receptor is capable of responding without T-cell help to epitope patterns meeting certain criteria, such as spacing of 5-10 $\mathrm{nm}$ between at least 10 repeating epitopes (10, 24-26).

For wildtype mice, rhIFN $\alpha 2 b$ is a foreign protein and it induces a classical immune response. All samples induced antibodies, although the level of antibodies induced by boiled rhIFNa2b was very low and antibodies were formed only in 1 mouse. The antibodies induced after immunization with glutaraldehyde treated rhIFN $\alpha 2 b$ did not cross-react with native rhIFN $\alpha 2 b$. This modified protein had apparently obtained new epitopes, due to the crosslinking, which are not present in native rhIFNo2b, as confirmed by Western blotting (figure 2c). However, the aggregates present in glutaraldehyde treated rhIFN $\alpha 2 \mathrm{~b}$ still contained native epitopes, but the level might be too low to elicit an immune response.

The observed poor immunogenicity of boiled rhIFN $\alpha 2 b$ was not expected but may be related to the size of the aggregates. The number of epitopes exposed to the immune system is inversely related to the size of the particles. Also the large size of the aggregates may slow down the clearance from the peritoneal cavity or impair the uptake by phagocytic cells. It was shown before that liposomes (average size $130 \mathrm{~nm}$ ) greatly decreased the clearance of 
encapsulated drugs out of the peritoneal cavity as compared to the free drug (27). The same authors also showed that the clearance was even more retarded when the liposomes were aggregated via avidin/biotin. The size of boiled rhIFN $\alpha 2 b$ could be well in the range of the aggregated liposomes.

In the transgenic mice immune tolerant for hIFNa2, antibodies to this protein can be induced either by breaking tolerance or via the classical immune response. Metal catalyzed oxidized rhIFN $\alpha 2 b$ was the only preparation capable of inducing antibodies in the transgenic mice. It is unlikely that the oxidation per sé explains this immunogenicity, because $\mathrm{H}_{2} \mathrm{O}_{2}$ oxidized rhIFN $\alpha 2 b$ lacked this effect while both products showed the same oxidation sites. It is more likely that the aggregate formation accompanying the metal catalyzed oxidation of rhIFN $\alpha 2 b$ was responsible for breaking the tolerance. The mechanism by which metal catalyzed oxidation induces aggregation of rhIFN $\alpha 2 b$ is unknown, but aggregation has also been reported for metal catalyzed oxidation of relaxin (18). It should also be noted that $\mathrm{H}_{2} \mathrm{O}_{2}$ oxidized rhIFN $\alpha 2 b$ and native rhIFN $\alpha 2 b$ contain dimers and apparently multimeric aggregation is needed to break tolerance.

Glutaraldehyde treated rhIFN $\alpha 2 b$ contained aggregates that were not able to break tolerance in the transgenic mice. The antibody response in the wildtype mice showed this sample to contain new epitopes for which the transgenics lack tolerance. Nevertheless, the transgenic mice did not respond. A reason for this could be that $\mathrm{T}$-cells recognized native linear epitopes, still present in the modified protein, for which the transgenic mice are immune tolerant. So the T-cells will not activate the B-cells into antibody production. In contrast with our results, Braun et al did show breaking of immune tolerance with glutaraldehyde induced aggregates (4). Reasons for this discrepancy could be differences in modification conditions, immunization protocol and/or route of administration.

Altogether, our data indicate that the dogma 'aggregation leads to immunogenicity of therapeutic proteins' should be taken with caution. The three different aggregated samples (obtained by metal catalyzed oxidation, by glutaraldehyde treatment, and by boiling), which differ both in size distribution and in protein structure, widely vary in immunogenicity: whereas aggregates obtained by metal catalyzed oxidation are able to break the immune tolerance, those obtained by glutaraldehyde treatment and boiling are not. If optimally spaced (5-10 $\mathrm{nm}$ ) repetitive (more than 10) native (or native like) epitopes are the predominant mechanism by which aggregates would break immune tolerance $(10,24-26)$, only the metal catalyzed oxidation product apparently fulfills these criteria. Also, the large aggregates detected by DLS and/or the larger aggregates detected by GPC, rather than the smaller 
oligomers observed by GPC, are most likely to be responsible for the increased immunogenicity of metal catalyzed oxidized rhIFN $\alpha 2 b$. Besides the number and the orientation of epitopes, however, the structure of the protein molecules that form the aggregates may be crucial. Both boiled rhIFN $\alpha 2 b$ (loss of native conformation) and glutaraldehyde treated rhIFN $\alpha 2 b$ (major chemical modifications) may not sufficiently resemble native rhIFN $\alpha 2 b$ to break the immune tolerance. So, aggregates composed of native-like protein molecules may be more likely to break tolerance than aggregates consisting of denatured proteins. This would imply that preventing the formation of native-like aggregates should be a major task for protein formulation scientists in their search for non-immunogenic therapeutic protein formulations.

\section{Conclusions}

In this study we tried to correlate structure with immunogenicity and ability to break immune tolerance. We found that large aggregates of denatured protein are not necessarily more immunogenic than smaller aggregates composed of more "native-like" protein. It appears that both the structure of the constituent proteins and/or the size of the aggregates determine immunogenicity. We also found that oxidation in itself does not enhance immunogenicity. Moreover, we have shown that screening the immunogenicity of protein drugs should include assays for antibodies to the modifications present in the product.

Although the aggregates produced in this study may not fully represent the degradation products typically formed during production, storage or handling of rhIFN $\alpha 2 b$ formulations, with the present study we gained more insight into which structural changes affect the immunogenicity of rhIFN $\alpha 2 b$. Clearly, more research is needed to clarify the relation between structure and immunogenicity of rhIFN $\alpha 2 b$ in particular and therapeutic proteins in general, to assess the minimum amount of modified proteins inducing antibody formation, and to evaluate the predictive value of immune tolerant transgenic mouse models for the immunogenicity of therapeutic proteins in patients. 


\section{Acknowledgments}

Dr. Pestka is kindly thanked for supplying the transgenic immune tolerant mice. Janny Westdijk is kindly acknowledged for her help with the Biacore experiments and valuable discussions. We thank Ronald van Ooijen and Georgina Gal for performing the mass spectrometric analyses.

\section{References}

1. G. Walsh. Pharmaceutical biotechnology products approved within the European Union. Eur J Pharm Biopharm 55: 3-10 (2003).

2. S. Hermeling, D. J. A. Crommelin, H. Schellekens and W. Jiskoot. Structure-immunogenicity relationships of therapeutic proteins. Pharm Res 21: 897-903 (2004).

3. H. Schellekens. Bioequivalence and the immunogenicity of biopharmaceuticals. Nat Rev Drug Discov 1: 457-462 (2002).

4. A. Braun, L. Kwee, M. A. Labow and J. Alsenz. Protein aggregates seem to play a key role among the parameters influencing the antigenicity of interferon alpha (IFN-alpha) in normal and transgenic mice. Pharm Res 14: 1472-1478 (1997).

5. E. Hochuli. Interferon immunogenicity: technical evaluation of interferon-alpha 2a. J Interferon Cytokine Res 17 Suppl 1: S15-21 (1997).

6. G. Schernthaner. Immunogenicity and allergenic potential of animal and human insulins. Diabetes Care 16 Suppl 3: 155-165 (1993).

7. W. V. Moore and P. Leppert. Role of aggregated human growth hormone (hGH) in development of antibodies to hGH. J Clin Endocrinol Metab 51: 691-697 (1980).

8. D. C. Robbins, S. M. Cooper, S. E. Fineberg and P. M. Mead. Antibodies to covalent aggregates of insulin in blood of insulin-using diabetic patients. Diabetes 36: 838-841 (1987).

9. B. Chackerian, D. R. Lowy and J. T. Schiller. Conjugation of a selfantigen to papillomavirus-like particles allows for efficient induction of protective autoantibodies. J Clin Invest 108: 415-423 (2001).

10. M. F. Bachmann, H. Hengartner and R. M. Zinkernagel. T helper cellindependent neutralizing $\mathrm{B}$ cell response against vesicular stomatitis virus: role of antigen patterns in B cell induction? Eur J Immunol 25: 3445-3451 (1995).

11. T. Fehr, M. F. Bachmann, H. Bluethmann, H. Kikutani, H. Hengartner and R. M. Zinkernagel. T-independent activation of B cells by vesicular 
stomatitis virus: no evidence for the need of a second signal. Cell Immunol 168: 184-192 (1996).

12. J. L. Ottesen, P. Nilsson, J. Jami, D. Weilguny, M. Duhrkop, D. Bucchini, S. Havelund and J. M. Fogh. The potential immunogenicity of human insulin and insulin analogues evaluated in a transgenic mouse model. Diabetologia 37: 1178-1185 (1994).

13. L. M. Pfeffer, C. A. Dinarello, R. B. Herberman, B. R. Williams, E. C. Borden, R. Bordens, M. R. Walter, T. L. Nagabhushan, P. P. Trotta and S. Pestka. Biological properties of recombinant alpha-interferons: 40th anniversary of the discovery of interferons. Cancer Res 58: 2489-2499 (1998).

14. P. Bonetti, G. Diodati, C. Drago, C. Casarin, S. Scaccabarozzi, G. Realdi, A. Ruol and A. Alberti. Interferon antibodies in patients with chronic hepatitic $\mathrm{C}$ virus infection treated with recombinant interferon alpha-2 alpha. J Hepatol 20: 416-420 (1994).

15. A. V. Palleroni, A. Aglione, M. Labow, M. J. Brunda, S. Pestka, F. Sinigaglia, G. Garotta, J. Alsenz and A. Braun. Interferon immunogenicity: preclinical evaluation of interferon-alpha 2a. $J$ Interferon Cytokine Res 17 Suppl 1: S23-27 (1997).

16. J. C. Ryff. Clinical investigation of the immunogenicity of interferonalpha 2a. J Interferon Cytokine Res 17 Suppl 1: S29-33 (1997).

17. Interferon alpha2b. European Pharmacopeia 5.o Vol 2: 1812-1815 (2004).

18. S. Li, T. H. Nguyen, C. Schoneich and R. T. Borchardt. Aggregation and precipitation of human relaxin induced by metal-catalyzed oxidation. Biochemistry 34: 5762-5772 (1995).

19. G. L. Peterson. A simplification of the protein assay method of Lowry et al. which is more generally applicable. Anal Biochem 83: 346-356 (1977).

20. ExPASy. Peptide Mass. http://ca.expasy.org/tools/peptide-mass.html (accessed 12 December 2004)

21. L. A. Kueltzo and C. R. Midddaugh. Ultraviolet Absorption spectroscopy. In W. Jiskoot and D. J. A. Crommelin (eds), Methods for structural analysis of protein pharmaeuticals, Vol. III, Biotechnology: Pharmaceutical Aspects, AAPS Press, Arlington, 2005, pp. 1-25.

22. E. T. J. Van den Bremer and A. J. R. Heck. Mass spectrometry: Protein conformational analysis and molecular recognition. In W. Jiskoot and D. J. A. Crommelin (eds), Methods for structural analysis of protein pharmaceuticals, Vol. III, Biotechnology: Pharmaceutical Aspects, AAPS Press, Arlington, 2005, pp. 435-464. 
23. ExPASy. ExPASy Proteomics Server. http://ca.expasy.org/uniprot/ Q86UP4 (accessed 1 June 2005)

24. B. Chackerian, P. Lenz, D. R. Lowy and J. T. Schiller. Determinants of autoantibody induction by conjugated papillomavirus virus-like particles. J Immunol 169: 6120-6126 (2002).

25. T. Fehr, M. F. Bachmann, E. Bucher, U. Kalinke, F. E. Di Padova, A. B. Lang, H. Hengartner and R. M. Zinkernagel. Role of repetitive antigen patterns for induction of antibodies against antibodies. J Exp Med 185: 1785-1792 (1997).

26. M. F. Bachmann, U. H. Rohrer, T. M. Kundig, K. Burki, H. Hengartner and R. M. Zinkernagel. The influence of antigen organization on B cell responsiveness. Science 262: 1448-1451 (1993).

27. W. T. Phillips, L. A. Medina, R. Klipper and B. Goins. A novel approach for the increased delivery of pharmaceutical agents to peritoneum and associated lymph nodes. J Pharmacol Exp Ther 303: 11-16 (2002). 
